\title{
Identificação e mapeamento das ações de enfermagem prescritas para pacientes internados em uma UTI de adultos
}

\author{
Identification and mapping of prescribed nursing actions for patients in an adult ICU
}

Identificación y asignación de acciones de enfermería prescritas para pacientes internados en una UTI de adultos

\section{Patricia Oliveira Salgado', Meire Chucre Tannure", Cleydson Rodrigues Oliveira"l', Tânia Couto Machado Chianca ${ }^{\text {IV }}$}

\author{
' Hospital Municipal Odilon Behrens, Coordenação de Especialidade e Ensino de Enfermagem. Belo Horizonte-MG, Brasil. \\ "Pontificia Universidade Católica de Minas Gerais, Campus Coração Eucarístico, \\ Curso de Enfermagem. Belo Horizonte-MG, Brasil. \\ "' Universidade Federal de Minas Gerais, Escola de Enfermagem, Programa de Pós-Graduação em Enfermagem. \\ (Mestrando). Belo Horizonte-MG, Brasil. \\ Iv Universidade Federal de Minas Gerais, Escola de Enfermagem, \\ Programa de Pós-Graduação em Enfermagem. Belo Horizonte-MG, Brasil.
}

\section{Submissão: 21-11-2010 Aprovação: 11-04-2012}

\section{RESUMO}

Estudo descritivo cujos objetivos foram identificar as ações de enfermagem prescritas por enfermeiros nos prontuários de pacientes internados em uma Unidade de Terapia Intensiva (UTI) Adulto de Belo Horizonte (MG), os termos empregados, sua frequência e mapear as ações à Teoria das Necessidades Humanas Básicas e às intervenções NIC._Obteve-se uma amostra de 44 prontuários. Identificou-se 2.260 ações de enfermagem. Após exclusão das repetições, encontrou-se 124 diferentes ações. Todas as ações de enfermagem foram mapeadas às necessidades psicobiológicas e também às intervenções NIC. Obteve-se $100 \%$ de concordância entre os expertos no processo de validação do mapeamento. Sugere-se que estudos semelhantes em UTI's de outras localidades e diferentes contextos/especialidades sejam conduzidos para identificar as ações de enfermagem elaboradas e sua evolução.

Descritores: Enfermagem; Cuidados de enfermagem; Classificação; Unidades de Terapia Intensiva; Adulto.

\section{ABSTRACT}

Descriptive study that aimed to identify nursing actions prescribed by nurses in the medical records of patients admitted to an Intensive Care Unit (ICU) for adults, in Belo Horizonte (MG), the terms used, their frequency and map the actions to the Theory of Basic Human Needs and NIC interventions. It was obtained a sample of 44 patient records. It was identified 2,260 nursing actions. After exclusion of repetitions, it was found 124 different actions. All nursing actions have been mapped to physiological needs and also to NIC interventions. It was obtained $100 \%$ of agreement among experts in the validation of the mapping process. It is suggested that similar studies in ICUs from other locations and different contexts / specialties should be driven to identify nursing actions developed and its evolution.

Key words: Nursing; Nursing care; Classification; Intensive Care Units; Adult.

\section{RESUMEN}

Estudio descriptivo que tuvo como objetivo identificar las acciones de enfermería prescritas por las enfermeras en las historias clínicas de pacientes ingresados en una Unidad de Cuidados Intensivos (UCI) de adultos de Belo Horizonte (MG), los términos utilizados, la frecuencia y el mapeo de las acciones con la Teoría de las Necesidades Humanas básica y las intervenciones NIC. Se obtuvo una muestra de 44 historias clínicas de pacientes. Se identificaron 2.260 acciones de enfermería. Tras la exclusión de las repeticiones, fueran encontradas 124 acciones diferentes. Todas las acciones de enfermería se han asignado a las necesidades fisiológicas y también a las intervenciones de la NIC. Se obtuvo 100\% de acuerdo entre los expertos en el proceso de validación de la asignación. Se sugiere que estudios similares sean desenvueltos en las UCI de otros lugares y diferentes contextos / especialidades, para identificar las acciones de enfermería desarrollados y su evolución.

Palabras clave: Enfermería; Atención de enfermería; Clasificación; Unidades de Terapia Intensiva, Adulto.

Extraído de Dissertação de Mestrado apresentada à Universidade Federal de Minas Gerais, Programa de Pós-Graduação em Enfermagem, 2010.

\section{AUTOR CORRESPONDENTE Patricia Oliveira Salgado E-mail: patriciaoliveirasalgado@gmail.com}




\section{INTRODUÇÃO}

O cuidado de enfermagem é considerado essencial para o tratamento dos pacientes, entretanto, ainda não é muito visível ou reconhecido. Isso vem acontecendo, dentre outros motivos, porque na prática assistencial ainda existe necessidade de se instrumentalizar os enfermeiros para implementar o Processo de Enfermagem de uma forma mais efetiva e com ações sistematizadas.

Essa realidade tem maior impacto, sobretudo nas Unidades de Tratamento Intensivo (UTI) pelo fato de serem necessárias avaliações críticas e rápidas dos pacientes, planos de cuidados abrangentes, serviços bem coordenados com outros profissionais da saúde, além de um efetivo e conveniente planejamento para a alta ${ }^{(1)}$.

A fim de contribuir com a implementação de ações seguras para os pacientes e conferir maior visibilidade para os cuidados de enfermagem, a padronização da linguagem de problemas e tratamentos de enfermagem tem sido desenvolvida para esclarecer e comunicar algumas regras essenciais na implementação dos cuidados ${ }^{(2)}$. Vale destacar que o Processo de Enfermagem é o principal recurso que os enfermeiros possuem para registrar o seu trabalho, avaliar a qualidade de suas atividades, aplicar e evidenciar seu conhecimento na assistência ao paciente e de consolidar sua prática profissional ${ }^{(3)}$.

Apesar deste esforço, ainda existem cuidados de enfermagem executados na prática que nem são padronizados, ou considerados efetivos para a melhora da condição clinica dos pacientes. Nestas situações os enfermeiros precisam dispor de habilidades técnicas e conhecimentos adquiridos com a experiência para examinar as tendências de sua prática, implementar os procedimentos e avaliar a qualidade de cuidados prestados aos pacientes. Contudo, tais ações precisam ser descritas, pesquisadas, divulgadas e validadas.

A descrição das ações de enfermagem prescritas para pacientes internados em UTI pode fornecer um perfil das necessidades de cuidados desses doentes, favorecer a organização do conhecimento sobre esses cuidados, além de permitir comparações entre populações com demandas específicas.

Assim, visando colaborar com a descrição dos cuidados de enfermagem prescritos por enfermeiros em UTIs de adultos, o presente estudo teve por objetivo identificar as ações de enfermagem prescritas por enfermeiros nos prontuários de pacientes internados em uma UTI de adultos de Belo Horizonte (MG), os termos empregados, sua frequência e mapear as ações à Teoria das Necessidades Humanas Básicas (NHB) e às intervenções NIC.

Justifica-se este estudo por considerar-se que a utilização de um conjunto de ações de enfermagem pode representar importante instrumento para a implementação da Sistematização da Assistência de Enfermagem (SAE) nas unidades onde a enfermagem presta assistência, em especial na UTI.

\section{MÉTODOS}

Trata-se de estudo descritivo desenvolvido em uma UTI de Adultos de Belo Horizonte (MG) destinada à internação particular ou por convênios. Para a realização deste estudo considerou-se como população todos os prontuários dos pacientes internados na UTI no período de 1 de setembro de 2008 a 30 setembro de 2009, totalizando 494 prontuários. Para estimar a representatividade das ações de enfermagem amostradas em relação aos 494 pacientes foi utilizado o método de reamostragem por permutação ${ }^{(4)}$. A amostra foi constituída por 44 prontuários que apesar de representar apenas $9 \%$ da população, possibilitaram estimar o percentual de ações de enfermagem prescritas.

Nos 44 prontuários foram coletadas 2.260 ações de enfermagem. Como havia neste conjunto prescrições de ações de enfermagem similares e repetidas, optou-se pela normalização de conteúdo com a realização da exclusão das repetições, correções de ortografia, análise de sinonímia, adequação de tempos verbais, uniformização de gênero e de número e exclusão das expressões pseudoterminológicas, que são definidas como elementos que ocorrem de forma casual no discurso, mas que não designam conceitos particulares, sendo considerado lixo terminológico ${ }^{(5)}$. Dessa forma, ao final desse processo restaram 124 diferentes ações de enfermagem.

Realizou-se o processo de mapeamento cruzado das 124 diferentes ações coletadas com a Teoria das $\mathrm{NHB}^{(6-7)}$ e as intervenções de enfermagem propostas pela $\mathrm{NIC}^{(2)}$, em sua $4^{\mathrm{a}}$ edição na versão traduzida para o português.

O mapeamento cruzado é um procedimento metodológico que liga palavras de sentido semelhante ou igual, por meio de um processo de tomada de decisão, usando estratégias indutivas ou dedutivas ${ }^{(8)}$. Pode ser utilizado na análise de dados, contidos no processo de enfermagem, nos diferentes campos do cuidado, através da comparação entre as informações existentes nos prontuários dos pacientes e as classificações de referência para a prática de enfermagem ${ }^{(9)}$.

As ações de enfermagem mapeadas à Teoria das NHB e às intervenções propostas pela NIC foram submetidas a um processo de validação, primeiramente por pesquisadores do Núcleo de Estudos e Pesquisas sobre Sistematizar o Cuidar em Enfermagem da Escola de Enfermagem da UFMG e, em seguida, foram encaminhadas para avaliação de enfermeiros intensivistas e pesquisadores da área da SAE que concordaram em participar do estudo como expertos, assinando o Termo de Consentimento Livre e Esclarecido (TCLE).

Como estratégia de validação do processo de mapeamento realizado utilizou-se a técnica Delphi, método para obter o consenso de um grupo de expertos sobre um determinado tema ${ }^{(10)}$. Não há diretriz que estabeleça o adequado nível de consenso a ser obtido ${ }^{(11)}$, porém, recomenda-se a obtenção do nível mínimo de concordância de $70 \%$, na etapa final da Técnica Delphi ${ }^{(12-14)}$. Diante dessa recomendação, adotou-se o índice de 70\% como nível mínimo de consenso a ser obtido pelos expertos neste estudo.

No processo de validação das ações de enfermagem mapeadas foram utilizados três instrumentos: um primeiro instrumento com as orientações necessárias e com as definições das $\mathrm{NHB}^{(6)}$; um segundo elencando as ações de enfermagem coletadas e mapeadas à Teoria das NHB e um terceiro instrumento com as ações de enfermagem mapeadas às intervenções da NIC. 
Para a realização da validação das ações de enfermagem mapeadas foram convidados, por e-mail, onze enfermeiros. Ao receber o convite, todos os profissionais aceitaram participar e receberam por e-mail o material da primeira etapa de validação (TCLE, um instrumento com as orientações necessárias para a validação e com as definições das NHB, o instrumento com as ações de enfermagem mapeadas à Teoria das NHB e o envelope selado e endereçado para retorno do TCLE assinado). Contudo, dois dos enfermeiros não responderam a primeira etapa dentro do prazo determinado, sendo excluídos do estudo.

Após o retorno dos instrumentos validados na primeira etapa encaminhou-se aos enfermeiros um novo instrumento com as ações de enfermagem mapeadas às intervenções propostas pela NIC, consistindo esta a segunda etapa do processo de validação.

Ao final da segunda etapa somente 6 dos profissionais realizaram a validação de todos o dados enviados, constituindo, então, a amostra de expertos deste estudo.

O estudo obteve parecer favorável da diretoria da unidade onde se realizou a pesquisa e do Comitê de Ética em Pesquisa (COEP) da Universidade Federal de Minas Gerais (Parecer COEP $\left.n^{\circ} 315 / 09\right)$, sendo seguidas as recomendações da resolução 196/96.

\section{RESULTADOS}

Para os 44 pacientes foram prescritas 2.260 ações de enfermagem, com uma média de 51 ações prescritas por paciente durante toda a internação na unidade. Após o processo de normalização do conteúdo obteve-se 124 diferentes ações de enfermagem, entre as quais 19 (15\%) apresentaram frequência maior que $50 \%$ (Tabela 1 ).

$\mathrm{Na}$ análise constatou-se que $52 \%$ (64) das diferentes ações de enfermagem identificadas foram prescritas para, no máximo, $9 \%$ dos pacientes.

\section{Mapeamento das ações de enfermagem às Necessidades Humanas Básicas}

As 124 ações de enfermagem identificadas foram mapeadas às NHB, conforme demonstrado na Tabela 2. Constatou-se que todas as ações prescritas referem-se às necessidades psicobiológicas.

A necessidade de Segurança Física e Meio Ambiente apresentou o maior número de ações de enfermagem mapeadas (32 - 26\%), abrangendo, também, o maior número de ações de enfermagem prescritas por prontuários (799 - 35\%) entre as 2.260 prescritas. À NHB Integridade Física foram mapeadas

Tabela 1 - Ações de enfermagem prescritas com frequência maior que 50\%. Belo Horizonte-MG, 2010.

\begin{tabular}{|c|c|c|}
\hline Ações de enfermagem prescritas & $\mathbf{N}$ & $\%$ \\
\hline Realizar hidratação cutânea com hidratante corporal após o banho & 44 & 100 \\
\hline Realizar higiene oral de $8 / 8$ horas e quando necessário & 44 & 100 \\
\hline Monitorar continuamente níveis de saturação, padrão respiratório e FR & 44 & 100 \\
\hline Manter cabeceira elevada se o estado hemodinâmico permitir & 44 & 100 \\
\hline Datar os equipos e trocá-los a cada $72 \mathrm{~h}$ & 44 & 100 \\
\hline Realizar o rodízio de dedos com sensor de oximetria de $2 / 2$ horas & 39 & 89 \\
\hline Realizar banho no leito de $24 / 24 \mathrm{~h}$ & 38 & 86 \\
\hline Manter paciente monitorizado continuamente durante banho no leito & 34 & 77 \\
\hline Trocar curativo de acesso venoso periférico de $24 / 24$ h e se apresentar sujidade e/ou umidade & 32 & 73 \\
\hline Informar ao enfermeiro presença de sinais flogísticos em sítio de inserção de cateter venoso periférico e/ou central & 32 & 73 \\
\hline Desprezar diurese de $2 / 2$ horas & 30 & 68 \\
\hline Mensurar a diurese de $2 / 2$ horas & 30 & 68 \\
\hline Medir temperatura axilar $2 / 2 \mathrm{~h}$ & 30 & 68 \\
\hline Verificar permeabilidade de acesso venoso periférico & 30 & 68 \\
\hline Checar estase $6 / 6 \mathrm{~h}$ & 28 & 64 \\
\hline Trocar curativo de pressão intra-arterial a cada 24 horas e se apresentar sujidade e/ou umidade & 27 & 61 \\
\hline Realizar mudança de decúbito de $2 / 2 \mathrm{~h}$ & 24 & 55 \\
\hline Aferir e anotar pressão arterial de $2 / 2 \mathrm{~h}$ & 24 & 55 \\
\hline Trocar periodicamente a fixação de sonda nasoentérica e observar sinais de lesão & 22 & 50 \\
\hline
\end{tabular}


Tabela 2 - Necessidades Humanas Básicas utilizadas no mapeamento das ações de enfermagem prescritas. Belo Horizonte-MG, 2010.

\begin{tabular}{lll}
\hline \multicolumn{1}{c}{ Necessidades Humanas Psicobiológicas } \\
\hline NHB & \multicolumn{2}{c}{$\begin{array}{c}\text { Ações de enfermagem } \\
\text { mapeadas }\end{array}$} \\
\cline { 2 - 3 } & N & \% \\
\hline Segurança Física e Meio Ambiente & 32 & 26 \\
Integridade Física & 31 & 25 \\
Regulação Vascular & 24 & 19 \\
Eliminação & 22 & 18 \\
Alimentação & 6 & 5 \\
Cuidado Corporal & 5 & 4 \\
Oxigenação & 2 & 2 \\
Regulação Térmica & 1 & 1 \\
Regulação Neurológica & 1 & $\mathbf{1 0 0}$ \\
\hline Total & $\mathbf{1 2 4}$ & \\
\hline
\end{tabular}

31 (25\%) diferentes ações de enfermagem, com um total de 739 ações de enfermagem prescritas.

Verificou-se que à NHB de regulação vascular foram mapeadas $24(19 \%)$ ações de enfermagem diferentes, sendo o cuidado de enfermagem manter cabeceira elevada se o estado hemodinâmico permitir prescrito 132 vezes. À NHB Cuidado Corporal foram mapeadas 5 diferentes ações de enfermagem, compreendendo 430 cuidados de enfermagem prescritos. Apesar da necessidade de Eliminação ter sido mapeada à $22(18 \%)$ diferentes ações de enfermagem, o percentual de prescrição dessas correspondeu a apenas 5\%, entre os 2260 cuidados de enfermagem prescritos para os pacientes da amostra. Ações de enfermagem foram mapeadas às NHB de oxigenação, regulação térmica, alimentação e regulação neurológica. Entretanto, este grupo foi formado por $10(8 \%)$ ações de enfermagem diferentes, compreendendo 286 (13\%) dos cuidados prescritos.

\section{Mapeamento das ações de enfermagem às intervenções propostas pela NIC}

As 124 diferentes ações de enfermagem identificadas também foram mapeadas às intervenções propostas pela NIC. Todas as ações puderam ser ligadas às Intervenções NIC. No

\begin{tabular}{|c|c|}
\hline Ações de enfermagem prescritas & Intervenção NIC \\
\hline Aferir e anotar pressão arterial de $2 / 2 \mathrm{~h}$ & 6680: Monitoração de Sinais Vitais \\
\hline Checar estase $6 / 6 \mathrm{~h}$ & 1056: Alimentação por sonda enteral \\
\hline Datar os equipos e trocá-los a cada $72 \mathrm{~h}$ & 6540: Controle de infecção \\
\hline \multirow{2}{*}{ Desprezar diurese $2 / 2 \mathrm{~h}$} & 0590: Controle da Eliminação Urinária \\
\hline & 0610: Cuidados na Incontinência Urinária \\
\hline $\begin{array}{l}\text { Informar ao enfermeiro presença de sinais flogísticos em sítio de inserção de cateter } \\
\text { venoso periférico e/ou central }\end{array}$ & 6550: Proteção contra infecção \\
\hline Manter cabeceira elevada se o estado hemodinâmico permitir & 0840: Posicionamento \\
\hline Manter paciente monitorizado continuamente durante banho no leito & 6654: Supervisão: segurança \\
\hline Medir temperatura axilar $2 / 2 \mathrm{~h}$ & 6680: Monitoração de Sinais Vitais \\
\hline \multirow{2}{*}{ Mensurar a diurese de $2 / 2 \mathrm{~h}$} & 0590: Controle da Eliminação Urinária \\
\hline & 0610: Cuidados na Incontinência Urinária \\
\hline \multirow{2}{*}{ Monitorar continuamente níveis de saturação, padrão respiratório e freqüência respiratória } & 3350: Monitoração respiratória \\
\hline & 3140: Controle de vias aéreas \\
\hline Realizar banho no leito de $24 / 24 \mathrm{~h}$ & 1610: Banho \\
\hline Realizar hidratação cutânea com hidratante corporal após o banho & 1610: Banho \\
\hline Realizar higiene oral de 8/8 horas e quando necessário & 1710: Manutenção da saúde oral \\
\hline \multirow{4}{*}{ Realizar mudança de decúbito de $2 / 2 \mathrm{~h}$} & 0840: Posicionamento \\
\hline & 0740: Cuidados com o repouso no leito \\
\hline & 3540: Prevenção de úlcera de pressão \\
\hline & 3520: Cuidados com Úlceras de Pressão \\
\hline Realizar o rodízio de dedos com sensor de oximetria de $2 / 2 \mathrm{~h}$ & 3584: Cuidados com a pele: tratamentos tópicos \\
\hline Trocar curativo de acesso venoso periférico de $24 / 24 \mathrm{~h}$ e se apresentar sujidade e/ou umidade & 6540: Controle de infecção \\
\hline Trocar curativo de PIA a cada $24 \mathrm{~h}$ e se apresentar sujidade e/ou umidade & 6540: Controle de infecção \\
\hline Trocar periodicamente a fixação de sonda nasoentérica e observar sinais de lesão & 1874: Cuidados com Sondas: Gastrintestinal \\
\hline Verificar permeabilidade de acesso venoso periférico & 2440: Manutenção de dispositivos para Acesso Venoso \\
\hline
\end{tabular}

Quadro 1 - Mapeamento das ações de enfermagem às intervenções NIC. Belo Horizonte-MG, 2010. 
Quadro 1 é apresentado o mapeamento das ações de enfermagem prescritas que apresentaram frequência maior que $50 \%$ às intervenções NIC.

Constatou-se que as 124 ações de enfermagem prescritas para os 44 pacientes da amostra foram relacionadas a apenas $42(8 \%)$ intervenções propostas pela NIC, entre as 514 disponíveis.

A taxonomia NIC é composta por 7 domínios e 30 classes de intervenções. Observa-se que as ações de enfermagem prescritas para os pacientes cujos prontuários foram analisados estão contempladas em somente 3 dos domínios da NIC. Um maior número de intervenções (23-55\%) foi encontrado no domínio fisiológico complexo. Essas intervenções estão distribuídas nas classes controle de medicamentos, controle neurológico, controle respiratório, controle da pele/feridas, controle da perfusão tissular. Outras intervenções (14-33\%) encontram-se no domínio fisiológico básico, distribuídas nas classes controle da eliminação, controle da imobilidade, facilitação do autocuidado e suporte nutricional. No domínio segurança encontram-se 5 (12\%) intervenções, todas na classe controle de risco. Quanto às classes de intervenções da NIC verifica-se que as ações de enfermagem identificadas compreenderam somente $10(33 \%)$ dessas.

\section{Validação das ações de enfermagem mapeadas}

Todos os enfermeiros que compuseram a amostra atenderam os critérios de inclusão, apresentando pontuação igual ou superior a $5^{(15)}$. Todos os profissionais trabalham e residem em Belo Horizonte, MG e são do sexo feminino. A seguir será apresentada a distribuição dos profissionais segundo a pontuação obtida(15) (Tabela 3).

Tabela 3 - Pontuação dos expertos que participaram do processo de validação dos diagnósticos e intervenções de enfermagem. Belo Horizonte/MG. 2010.

\begin{tabular}{lcc}
\hline Pontuação & N & \% \\
\hline 5 a 7 pontos & 2 & 33 \\
8 a 10 pontos & 1 & 17 \\
11 a 13 pontos & 2 & 33 \\
14 pontos & 1 & 17 \\
Total & $\mathbf{6}$ & $\mathbf{1 0 0}$ \\
\hline
\end{tabular}

Verifica-se que os expertos deste estudo são qualificados, aliando além da experiência prática (a idade mínima de experiência prática foi de 4 anos) a experiência com atividades acadêmica e de pesquisa.

Para a validação das ações de enfermagem mapeadas junto aos enfermeiros expertos nas áreas de terapia intensiva e sistematização da assistência de enfermagem utilizou-se uma rodada da Técnica Delphi tanto para o mapeamento das ações de enfermagem às NHB quanto ao mapeamento às intervenções propostas pela NIC, uma vez que foi alcançado o nível mínimo de concordância estabelecido entre os avaliadores já na primeira rodada. Verificou-se que o grau de concordância entre o grupo de expertos no processo de validação das ações de enfermagem, foi de $100 \%$ de concordância (Kappa $=1,0)$.

\section{DISCUSSÃO}

Entre as ações de enfermagem identificadas constatou-se que $5(4 \%)$ foram prescritas para todos os pacientes. A partir do mapeamento das ações às NHB realizado verificou-se que todas as ações prescritas se relacionaram às necessidades psicobiológicas.

Compreende-se que, em função da gravidade do estado de saúde física dos pacientes internados em uma UTI, a equipe de enfermagem, ao prestar os cuidados a este tipo de paciente, priorize a assistência às necessidades psicobiológicas afetadas. Entretanto, tanto para o paciente como para a família do paciente a sua internação em uma UTI pode ser um sinal de ameaça à vida e ao bem-estar. Ela é marcada por várias modificações no hábito de vida, como a separação dos familiares, substituição do cuidado pela equipe intensivista e ainda a exposição a um ambiente desconhecido, com movimentação constante de profissionais, repleto de aparelhos com ruídos e alarmes, iluminação constante, variação térmica e a realização frequente de vários procedimentos invasivos.

As necessidades humanas são inter-relacionadas e fazem parte de um todo indivisível do ser humano de tal forma que, quando uma se manifesta todas elas sofrem algum grau de alteração $^{(7)}$. Assim, as prioridades para a assistência de enfermagem têm de ser ajustadas sistematicamente, e considerar a integralidade do ser humano.

No mapeamento das ações prescritas pelos enfermeiros às intervenções NIC, foi identificado que todas fazem parte da classificação. Entende-se, portanto, que as intervenções NIC contemplam as ações de enfermagem prescritas, representando a prática de enfermagem no contexto desta UTI. Das 124 ações de enfermagem, 37 (30\%) foram mapeadas a mais de uma intervenção NIC. Este fato pode ser explicado devido a diferenças na terminologia usada nas prescrições estabelecidas pelos enfermeiros e na $\mathrm{NIC}^{(16)}$. Esta realidade pode levar, em alguns casos, a mais de uma interpretação para o que está sendo mapeado. Além disso, cabe ressaltar que na classificação há atividades semelhantes para diferentes intervenções.

Quando são analisadas as diferentes intervenções NIC utilizadas no mapeamento cruzado e sua relação com os domínios dessa classificação, verifica-se que essas compreenderam somente 3 dos 7 domínios, sendo que a maioria está localizada no domínio fisiológico complexo 25 (55\%), seguida pelo domínio fisiológico básico 15 (33\%) e o domínio segurança 5 (12\%).

Em um trabalho que também mapeou as ações de enfermagem prescritas para pacientes internados em UTI de Adultos às intervenções NIC encontrou-se, também, a maioria das intervenções localizadas no domínio fisiológico complexo seguido pelo fisiológico básico, com percentual de $42 \%$ e $37 \%$, respectivamente ${ }^{(16)}$.

Em relação às classes de cada domínio, o predomínio das intervenções está localizado na classe $\mathrm{K}$ - controle respiratório, e na classe $\mathrm{N}$ - controle da perfusão tissular, ambas com 
8 intervenções NIC. Esse dado remete ao número elevado de pacientes internados nesta UTI com problemas de saúde relacionados ao sistema respiratório e à perfusão tissular, necessitando, portanto, de cuidados para promover a desobstrução de vias aéreas e a troca de gases eficazes, e de intervenções para otimizar a circulação do sangue e líquidos aos tecidos.

Analisando o perfil dos enfermeiros que validaram as ações de enfermagem mapeadas foi possível observar que $83 \%$ apresentam prática clínica de pelo menos um ano de duração na área de terapia intensiva, com a utilização de diagnósticos, intervenções ou resultados de Enfermagem na prática clínica e todos apresentam especialização em terapia intensiva. Ressalta-se, ainda, o elevado número de expertos (5-83\%) que possui publicações na área de terapia intensiva, diagnósticos, intervenções ou resultados de enfermagem.

Obteve-se $100 \%$ de concordância (Kappa $=1,0$ ) entre os expertos na avaliação das ações de enfermagem mapeadas às NHB e, também, na avaliação das ações que foram mapeadas às Intervenções NIC em uma única rodada da Técnica Delphi. Tal fato pode ser explicado devido a primeira etapa do mapeamento cruzado realizado ter sido submetido a um processo de análise e confirmação por um grupo de pesquisadores na área de SAE e, por ter sido realizado na segunda etapa um processo de validação das ações de enfermagem confirmadas na primeira etapa por enfermeiros expertos.

\section{CONCLUSÕES}

Este estudo permitiu identificar as ações enfermagem mais frequentes prescritas para os pacientes internados em uma UTI de Adultos. Para os 44 pacientes cujos prontuários foram analisados encontrou-se 124 diferentes ações de enfermagem prescritas. Dessas, apenas 5 foram prescritas para $100 \%$ dos pacientes.

Todas as ações de enfermagem identificadas foram mapeadas às necessidades psicobiológicas. Quanto ao processo de mapeamento às intervenções NIC executado, foi possível fazer a ligação de $100 \%$ das ações às intervenções sugeridas na NIC.

$\mathrm{O}$ estudo realizado permitiu verificar que a classificação NIC tem um conteúdo de intervenções abrangentes que podem favorecer na elaboração dos planos de cuidados de enfermagem. Com o auxílio dessa classificação, os enfermeiros podem prescrever cuidados de forma elaborada tanto em profundidade como em abrangência, visando favorecer uma melhor documentação e a comunicação entre os profissionais e consequentemente contribuir para o desenvolvimento da área.

Como a enfermagem tem buscado a integralidade no cuidado que presta, sugere-se que estudos semelhantes em UTI de outras localidades e diferentes contextos/especialidades sejam conduzidos no sentido de acompanhar a evolução das prescrições de enfermagem em relação a identificação e atendimento a outras NHB para além das necessidades psicobiológicas.

\section{REFERÊNCIAS}

1. Nascimento ERP do, Trentini M. O cuidado de enfermagem na unidade de terapia intensiva (UTI): teoria humanística de Paterson e Zderad. Rev Latinoam Enferm [periódico na Internet]. 2004 Abr [acesso em 14 jun 2010];12(2):250-7 Disponível em: http://www.scielo.br/scielo.php?script =sci_ arttext\&pid $=$ S0104-11692004000200015\&lng $=$ pt.

2. Dochterman JM, Bulechek GM. Classificação das intervenções de enfermagem (NIC). 4.ed. Porto Alegre: Artmed; 2008.

3. Lima CLH, Nóbrega MML. Nomenclatura de intervenções de enfermagem para clínica médica de um hospital escola. Rev Bras Enferm 2009;62(4):570-8.

4. Good PI. Permutation tests: a pratical guide to resampling methods for testing hyphoteses. 2nd. ed. New York: Springer-Verlag; 1994. (Springer series in statistics).

5. Pavel S, Nolet D. Manual de terminologia. Canadá: Public Words and Govermment Services; 2001. p. 166.

6. Benedet AS, Bub MBC. Manual de diagnóstico de enfermagem: uma abordagem baseada na teoria das necessidades humanas básicas e na classificação diagnóstica da NANDA. 2. ed. Florianópolis: Bernúncia; 2001.

7. Horta WA. Enfermagem: teoria, conceitos, princípios e processo. Rev Esc Enferm USP 1974; 8(1):7-15.

8. Coenen A, Ryan P, Sutton J. Mapping nursing interventions from a hospital information system to the nursing interventions classification (NIC). Nurs Diagn 1997;8(4):145-51.
9. Moorhead S, Delaney C. Mapping nursing intervention data into the nursing interventions classification (NIC): process and rules. Nurs Diagn 1997;8(4):137-44.

10. Goodman CM. The Delphi Technique: a critique. J Adv Nurs 1987;12(3):729-34.

11. Keeney S, Hasson F, Mckenna $\mathrm{H}$. Consulting the oracle: ten lessons from the Delphi Technique in nursing research. J Adv Nurs 2006;2(53):205-12.

12. Grant JS, Kinney MR. Using the Delphi technique to examine content validity of nursing diagnosis. Nurs Diagn 1992;3(1):12-22.

13. Perroca MG, Gaidzinski RR. Sistema de classificação de pacientes: construção e validação de um instrumento. Rev Esc Enferm USP 1998;32(2):153-68.

14. Mancussi AC. Assistência ao binômio paciente/família na situação de lesão traumática da medula espinhal. Rev Latinoam Enferm 1998;6(4):67-73.

15. Fehring RJ. Methods to validate nursing diagnoses. Heart \& Lung 1987;16(6):625-9.

16. Lucena AF, Gutiérrez MGR, Echer IC, Barros ALBL. IntervenÇões de enfermagem utilizadas na prática clínica de uma unidade de terapia intensiva. Rev Latinoam Enferm [periódico na Internet]. 2010 Out [acesso 11 Abr 2012];18(5):873-880 Disponível em: http://www.scielo.br/scielo.php?script = sci arttext\&pid = S0104-11692010000500006\&lng = pt. doi:10.1590/S0104-11692010000500006. 